\title{
MORITA EQUIVALENCE OF SIMPLE NOETHERIAN RINGS
}

\author{
J. T. STAFFORD ${ }^{1}$
}

\begin{abstract}
We show that a simple Noetherian ring of finite global
\end{abstract} dimension and Krull dimension one is Morita equivalent to a domain.

In [4] is given an example of a simple Noetherian ring that is not Morita equivalent to a domain. Now the ring in question has infinite global dimension but Krull dimension one. In contrast, we show here that if $R$ is a simple Noetherian ring of finite global dimension and Krull dimension one, then $R$ is Morita equivalent to a domain. This is a corollary of Theorem 1, which gives a generalisation of this result to simple rings of arbitrary finite Krull dimension.

Throughout this note all rings will contain an identity and all modules will be unitary. We will denote the uniform dimension of a module $M$ by $\operatorname{ud}(M)$ and the projective dimension by $\operatorname{pd}(M)$. The left Krull dimension of a ring $R$ will be denoted by $l-\operatorname{Kdim}(R)$.

TheOREM 1. Let $R$ be a simple Noetherian ring of finite global dimension. Then $R$ is Morita equivalent to a ring $S$ such that

$$
\operatorname{ud}(S) \leqslant l-\operatorname{Kdim}(S) .
$$

Proof. Choose $S$ Morita equivalent to $R$ such that $S$ has the smallest possible uniform dimension. Suppose that $\operatorname{ud}(S)=r$ and $\operatorname{Kdim}(S)=n$. If $r \leqslant n$, then we are through, so suppose that $r \geqslant n+1$. Observe that, if $P$ is an indecomposable finitely generated projective right $S$-module, then

$$
r \leqslant \operatorname{ud}(P)<n+r<2 r,
$$

the first inequality following from the minimality of $S$ and the second by [3, Theorem 5.2]. Let $P_{1}, \ldots, P_{m}$ be finitely generated projective right $S$ modules such that:

(a) $\operatorname{ud}\left(P_{i}\right)=r+a_{i}$ where $0<a_{i}<r$;

(b) if $P$ is a finitely generated projective right $S$-module with $\operatorname{ud}(P)=r+$ $a$ for $0<a<r$, then $a=a_{i}$ for some $a_{i}$.

(If no such modules exist, so much the better.) Let $H$ be the additive subgroup of $\mathbf{Z} / r \mathbf{Z}$ generated by the $a_{i}$ 's. Note that, for all $h \neq 0 \in H, h=a_{i}$

Received by the editors June 14, 1978.

AMS (MOS) subject classifications (1970). Primary 16A12, 16A48.

'Supported by the British Science Research Council through a NATO Research Fellowship. 
for some $i$. For, suppose that

$$
h=a_{1} h_{1}+\cdots+a_{m} h_{m}-r f
$$

for some integers $h_{i}$ and $f$. Then, by [3, Theorem 5.2],

$$
P_{1}^{\left(h_{1}\right)} \oplus \cdots \oplus P_{m}^{\left(h_{m}\right)} \cong S^{(t)} \oplus Q,
$$

where $Q$ is a projective $S$-module with $\operatorname{ud}(Q) \leqslant 2 r$. Clearly $\operatorname{ud}(Q)=r+h$, as required.

Suppose that $H=\mathbf{Z} / r \mathbf{Z}$. Then there exists a projective $S$-module $Q$ with $n+r \leqslant \operatorname{ud}(Q)<2 r$. But then by [3, Theorem 5.2], $Q \cong S \oplus Q^{\prime}$ and $\operatorname{ud}\left(Q^{\prime}\right)$ $<r$, which contradicts the minimality of $\mathrm{ud}(S)$. So $H \neq \mathbf{Z} / r \mathbf{Z}$. Let $a$ be the minimal element of $H$ (if all finitely generated projective right $S$-modules have uniform dimension some multiple of that of $S$, put $a=r$ ). Then $a>1$ and $a$ divides $r$. Further, any finitely generated projective right $S$-module $P$ has $\operatorname{ud}(P)=m a$ for some integer $m$. The argument now closely follows that of [5, Lemma 2.8].

Let $t$ be an integer $\geqslant 1$. Suppose, given any finitely generated torsion-free right $S$-module $K$ with $\operatorname{pd}(K) \leqslant t-1$, that $\operatorname{ud}(K)$ is an integer multiple of $a$ (by the last paragraph this holds for $t=1$ ). Let $M$ be a finitely generated torsion-free right $S$-module with $\operatorname{pd}(M)=t$. Then there exists a short exact sequence

$$
0 \rightarrow K \rightarrow S^{(m)} \rightarrow M \rightarrow 0,
$$

for some integer $m$ and module $K$. Now $\operatorname{pd}(K)=t-1$ and so $\operatorname{ud}(K)=c a$ for some integer $c$. But, by [2, Lemma 3.6], uniform dimension is additive in this situation. So $\mathrm{ud}(M)=d a$ for some integer $d$. Hence by induction and the fact that $S$ has finite global dimension, every torsion free $S$-module has uniform dimension some integer multiple of $a$. But this implies that $a=1$, a contradiction.

COROLlaRY 2. Let $R$ be a simple Noetherian ring with finite global dimension and $l-\operatorname{Kdim}(R)=n<\infty$. If $\mathrm{ud}(R) \geqslant 2 n$, then $R$ contains nontrivial idempotents.

Proof. By the theorem, $R$ is Morita equivalent to a $\operatorname{ring} S$ with $\operatorname{ud}(S) \leqslant n$. Let $P$ be the image of $R$ under the equivalence of the right module categories. Then

$$
\operatorname{ud}(P)=\operatorname{ud}(R) \geqslant \operatorname{ud}(S)+n .
$$

So, by [3, Theorem 5.2], $P$ has a free direct summand. Hence $R=I \oplus J$ for some nonzero right ideals $I$ and $J$. Thus $R$ has nontrivial idempotents.

COROllary 3. Let $R$ be a simple Noetherian ring with finite global dimension and $l-\operatorname{Kdim}(R)=1$. Then $R$ is Morita equivalent to a domain. If $R$ is not a domain, then $R$ has nontrivial idempotents.

Note that, as is shown by the example in [4] and [6], all three results are false if $R$ has infinite global dimension. For rings of finite global dimension these corollaries give partial positive answers to [1, Questions 3 and 5, p. 381]. 


\section{REFERENCES}

1. C. Faith, Algebra: rings, modules and categories. I, Springer-Verlag, Berlin and New York, 1973.

2. A. W. Goldie, Rings with maximum condition, Mimeographed notes, Yale University, 1961.

3. J. T. Stafford, Stable structure of noncommutative Noetherian rings. II, J. Algebra 52 (1978), 218-235.

4. , A simple Noetherian ring not Morita equivalent to a domain, Proc. Amer. Math. Soc. 68 (1978), 159-160.

5. R. Walker, Local rings and normalizing sets of elements, Proc. London Math. Soc. (3) 24 (1972), 27-45.

6. A. E. Zalesskii and O. M. Neroslavskii, There exist simple Noetherian rings with zero divisors but without idempotents, Comm. Algebra 5 (1977), 231-244. (Russian)

Department of Mathematics, Brandeis University, Waltham, MassachusetTs 02154

Current address: Gonville and Caius College, Cambridge, England 